\title{
A Novel Faults Diagnosis Method for Rolling Element Bearings Based on EWT and Ambiguity Correlation Classifiers
}

\author{
Xingmeng Jiang ${ }^{1}, \mathrm{Li} \mathrm{Wu}^{2}$ and Mingtao $\mathrm{Ge}^{2, *}$ \\ 1 Department of Electronic Engineering, Zhengzhou Railway Vocational \& Technical College, No. 9 \\ Qiancheng Road, Zhengdong New District, Zhengzhou 451460, Henan, China; mexingjiang@126.com \\ 2 College of Electronics and Information Engineering, SIAS International University, No. 168 Renmin Road, \\ Xinzheng 451150, Henan, China; xqytju@163.com \\ * Correspondence: gemingtao@sias.edu.cn or stevenget@126.com; Tel.: +86-150-9338-0110
}

Academic Editors: Raúl Alcaraz Martínez and Kevin Knuth

Received: 20 March 2017; Accepted: 15 May 2017; Published: 18 May 2017

\begin{abstract}
According to non-stationary characteristic of the acoustic emission signal of rolling element bearings, a novel fault diagnosis method based on empirical wavelet transform (EWT) and ambiguity correlation classification (ACC) is proposed. In the proposed method, the acoustic emission signal acquired from a one-channel sensor is firstly decomposed using the EWT method, and then the mutual information of decomposed components and the original signal is computed and used to extract the noiseless component in order to obtain the reconstructed signal. Afterwards, the ambiguity correlation classifier, which has the advantages of ambiguity functions in the processing of the non-stationary signal, and the combining of correlation coefficients, is applied. Finally, multiple datasets of reconstructed signals for different operative conditions are fed to the ambiguity correlation classifier for training and testing. The proposed method was verified by experiments, and experimental results have shown that the proposed method can effectively diagnose three different operative conditions of rolling element bearings with higher detection rates than support vector machine and back-propagation (BP) neural network algorithms.
\end{abstract}

Keywords: ambiguity correlation classifier; empirical wavelet transform; faults diagnosis; rolling element bearings

\section{Introduction}

The rolling element bearing represents one of the most important elements in the industrial equipment field, and they are widely used in many important equipment fields, such as space flight and aviation, wind power generation, etc. The operative conditions of rolling bearings play a vital role in the proper operation of equipment [1-3]. However, complex working environments and human factors might cause bearing faults or, in the worst case, major accidents [4]. Thus, it is necessary to diagnose the operative condition of rolling element bearings in order to provide a plan for subsequent repairs and to guarantee a proper operation of industrial equipment.

Nowadays, the monitoring of status of rolling element bearings is performed by the processing of collected stress signals, acoustic emission signals (AE), vibration analysis, and current signals. The traditional method for vibration analysis detection has been widely used in bearing fault diagnosis, but no significant changes occur in the vibration signal in the case of an early-stage fault. It is difficult to clearly identify the fault due to the easy mixture between the vibration signal from other components of the mechanical equipment, the fault signal, and the interference from the surrounding noise. Bearing fault detection based on the current signal uses the stator current signal analysis method. 
It is characterized by non-invasive detection. The method is successfully applied in bearing fault detection. The stator current signal is more convenient, simpler, and more practical in extraction than the vibration signal. However, the stator current signal is susceptible to the interference from the fundamental frequency and noise, thus making it difficult to extract the fault characteristic quantity as it contains a small fault-related frequency component. The acoustic emission signal has a wide frequency spectrum ranging from several $\mathrm{kHz}$ to $\mathrm{MHz}$. Using the acoustic emission signal for diagnosis of the bearing fault can easily avoid the interference from the low-frequency vibration signal and achieve high sensitivity and real-time monitoring. Morhain and Mba undertook an investigation to ascertain the most appropriate threshold level for AE count diagnosis in rolling element bearings [5]. A comparative study on the use of AE and vibration analysis was conducted, which concluded that AE offers earlier fault detection and improved identification capabilities than vibration analysis. Furthermore, the $\mathrm{AE}$ technique also provided an indication of the defect size, allowing the user to monitor the rate of degradation of the bearing.

Moreover, the acoustic emission method has attracted wide attention from scholars because of its high sensitivity and availability in real-time on-line monitoring [6,7]. Accordingly, an acoustic emission method is used to monitor different operative conditions of rolling bearings in this paper $[8,9]$. In this study, the bearing faults were seeded on the rolling elements and on the inner and outer races, and each faulty bearing was reinstalled (separately) on the test rig. Bearing fault diagnosis has been studied in the past decades and promising results have been offered in a large volume of prior literature [10]. Many researchers have discussed the spectral analysis methodologies for bearing fault diagnosis [11,12]. A vibration spectrum was referred to as a mechanical signature, and the determination of information from it was referred to as signature analysis. Traditionally, mechanical signatures were obtained standardly from known healthy machines. Signatures taken at later occasions could be compared according to the noted standard and significant changes, which leads to corrective actions. Ideally, the signature components or contributions can be attributed to specific causes (i.e., mechanical parts), in order to facilitate the specific remedial actions [13]. Therefore, the impending failures can be predicted. Even though the spectral analysis methods have demonstrated the capability of diagnosing bearing defects and have shown promising results, the bearing fault-related features are generally clouded by massive uncorrelated signals; thus, it is not easy to observe the fault features of bearings when the previous methods are used. In the case of rolling element bearings, the acoustic emission technique has been extensively studied by researchers. When faults occur in the bearing, impulse mode components caused by the faults will appear in the acoustic emission signal. The acoustic emission signals consist of fault components related to the system dynamical characteristics. However, due to factors of nonlinear stiffness and clearance of the bearing, the acoustic emission signals often exhibit as non-stationary. A non-stationary signal processing method must be employed to obtain mode decomposition from the fault signal.

The traditional non-stationary signal analysis method and wavelet decomposition method cannot obtain effective feature information due to a lack of adaptability [14-16]. The empirical mode decomposition (EMD) represents a classical time-frequency analysis method. However, the decomposed (IMF) component loses its true physical significance due to mode mixing in the EMD decomposition process [17-19]. In order to solve this issue, the empirical wavelet transform (EWT) is proposed to construct the wavelet basis in an adaptive way [20,21]. In contrast to EMD decomposition, EWT processes the signal within the wavelet framework, has a sufficient theoretical basis, and can extract an inherent mode of the signal $[22,23]$. Based on wavelet theory, the mode mixing problem can be resolved using EWT. Moreover, EWT has more applications than EMD. Therefore, it is worth introducing EWT into the rolling element bearing fault to provide better detection performance than the performance obtained by EMD-based methods [24,25]. The acoustic emission signals consist of fault information related to the rolling element bearings. The EWT method is used to analyze the acoustic emission signal and to obtain the mode components; some of decomposed components were sensitive to fault information, while others were not correlated with faults. Thus, these components 
needed to be processed further in order to extract the related mode components of the fault and to reject the noise interference. The mutual information was a measure of the statistical correlation between two random variables. The higher the correlation between the two random variables, the greater the mutual information. Thus, the related mode components of the fault were extracted using the mutual information. The traditional back-propagation (BP) neural network and the support vector machine (SVM) algorithms are widely used for fault diagnosis. Riahi et al. distinguished signals of different corrosion stages with a BP neural network in acoustic emission testing of a tank bottom [26,27]. However, a BP neural network has the disadvantages of complex parameter setting, slow convergence, falling easily into local minima, and limited accuracy and scope of application [28]. Compared to a BP neural network, SVM generalization performance is better, but it still requires manual assignment of kernel functions and kernel function parameters [29,30], which limits the significance of SVM applications.

Based on the above analysis, a novel fault detection method for rolling element bearings based on empirical wavelet transform and ambiguity correlation classification (ACC) is proposed. The EWT method is used to analyze the vibration signal and to obtain the accurate mode components without mode mixing generated in the EMD method. In addition, a mutual-information selection method is proposed to select the de-noised components of the vibration signals and to reconstruct the signal. In order to address the disadvantages of traditional BP and SVM classifiers, such as complex parameter setting and low convergence rate, the ambiguity functions and the correlation coefficients are combined to achieve the ambiguity correlation classification. The reconstructed signal is used as an input of the ACC intended for training and testing in order to provide the monitoring of operative conditions of rolling element bearings.

This paper is organized as follows: In Section 2, the EWT method is explained and illustrated by simulations. In Section 3, the proposed classification based on ambiguity correlation is presented in detail. In Section 4, the fault diagnosis method based on EWT and ACC is introduced. The proposed fault-detection scheme is experimentally validated and compared to the diagnosis method based on EMD in Section 5. Lastly, the conclusions of the paper are given in Section 6.

\section{EWT Decomposition Method}

\subsection{EWT Principle}

The empirical wavelet transform represents a self-adaptive signal processing method proposed by Gilles et al. in 2013 [20-22]. Namely, EWT can obtain a series of frequency modulation signals and amplitude modulation signals by self-adaptive segmentation of the signal frequency spectrum. The mathematical expression of complex decomposed signal $f(t)$ is:

$$
f(t)=\sum_{k=0}^{N} f_{k}(t)
$$

where $f_{k}(t)$ denotes the decomposed component, and $f_{k}(t)$ stands for the frequency-modulated amplitude-modulated signal, $f(t)$ is a complex decomposed signal.

The empirical wavelet transform provides the wavelet filter bank by self-adaptive segmentation of the Fourier spectrum of the signal. In this method, it is firstly supposed that Fourier supports segmentation of $N$ continuous parts in a segmentation interval $\Lambda_{n}=\left[\omega_{n-1}, \omega_{n}\right],\left(\omega_{0}=0, \omega_{n}=\pi\right)$, where $\omega_{n}$ represents the boundary between different parts, and a $\cup_{n=1}^{N} \Lambda_{n}=[0, \pi]$ partitioned graph is a transitional segment with the center $\omega_{n}$ and width of $2 \tau_{n}$. When the segmentation interval $\Lambda_{n}$ is determined, the empirical wavelet defines the band-pass filter on each section of $\Lambda_{n}$. Based on this concept, Gilles reconstructed an empirical wavelet using the Meyer wavelet reconstruction method. 
For $n$ greater than 0 , the empirical scaling function $\widehat{\phi}_{n}(\omega)$ and the empirical wavelet function $\hat{\varphi}_{n}(\omega)$ can be expressed as:

$$
\begin{gathered}
\hat{\phi}_{n}(\omega)\left\{\begin{array}{l}
1,|\omega| \leq(1-\gamma) \omega_{n} \\
\cos \left[\frac{\pi}{2} \beta\left(\frac{1}{2 \gamma \omega_{n}}(|\omega|)\right)\right],(1-\gamma) \omega_{n} \leq|\omega| \leq(1+\gamma) \omega_{n} \\
0, \text { otherwise }
\end{array}\right. \\
\hat{\psi}_{n}(\omega)\left\{\begin{array}{l}
1,(1+\gamma) \omega_{n} \leq|\omega| \leq(1-\gamma) \omega_{n+1} \\
\cos \left[\frac{\pi}{2} \beta\left(\frac{1}{2 \gamma \omega_{n}}(|\omega|)\right)\right],(1-\gamma) \omega_{n} \leq|\omega| \leq(1+\gamma) \omega_{n} \\
\sin \left[\frac{\pi}{2} \beta\left(\frac{1}{2 \gamma \omega_{n}}\left(|\omega|-(1-\gamma) \omega_{n}\right)\right)\right],(1-\gamma) \omega_{n} \leq|\omega| \leq(1+\gamma) \omega_{n} \\
0, \text { otherwise }
\end{array}\right.
\end{gathered}
$$

where:

$$
\begin{gathered}
\tau_{n}=\gamma \omega_{n} \\
\beta(x)=x^{4}\left(35-84 x+70 x^{2}-20 x^{3}\right) \\
\gamma<\min \left(\frac{\omega_{n+1}-\omega_{n}}{\omega_{n+1}+\omega_{n}}\right)
\end{gathered}
$$

where $\widehat{\phi}_{n}(\omega)$ is the empirical scaling function, and $\hat{\varphi}_{n}(\omega)$ is the empirical wavelet function. The traditional wavelet transform is used to construct the EWT. Namely, it is supposed that $F[$.$] and F^{-1}[$. are the Fourier transformation and inverse Fourier transformation, respectively. The empirical wavelet high-frequency component is obtained from the inner product of the signal by the empirical wavelet function. The corresponding mathematical expression is as follows:

$$
\begin{aligned}
& W_{f}^{e}(n, t)=<f(t), \psi_{n}(t)>=\int f(\tau) \overline{\psi_{n}(\tau-t)} d \tau \\
& =F^{-1}[f(\omega) \hat{\psi}(\omega)]
\end{aligned}
$$

where $W_{f}^{e}(n, t)$ is the high-frequency component of the empirical wavelet. Then, the low-frequency component can also be obtained from the inner product of the signal by the empirical scale function:

$$
\begin{aligned}
& W_{f}^{e}(0, t)=<f(t), \phi_{1}(t)>=\int f(\tau) \overline{\phi_{1}(\tau-t)} d \tau \\
& =F^{-1}\left[f(\omega) \hat{\phi}_{1}(\omega)\right]
\end{aligned}
$$

where $W_{f}^{e}(0, t)$ is the low-frequency component of the empirical wavelet. Finally, the reconstructed original signal is obtained from high-frequency and low-frequency components:

$$
\begin{aligned}
& f(t)=W_{f}^{e}(0, t) \times \phi_{1}(t)+\sum_{n=1}^{N} W_{f}^{e}(n, t) \times \psi_{n}(t) \\
& =F^{-1}\left[\hat{W}_{f}^{e}(0, \omega) \hat{\phi}_{1}(t)+\sum_{n=1}^{N} \hat{W}_{f}^{e}(n, \omega) \times \hat{\psi}_{n}(\omega)\right]
\end{aligned}
$$

where $\hat{W}_{f}^{e}(0, \omega)$ and $\hat{W}_{f}^{e}(n, \omega)$ are the Fourier transformations of $W_{f}^{e}(0, t)$ and $W_{f}^{e}(n, t)$, respectively. Thus, the mathematical expressions of the frequency-modulated amplitude-modulated signal are as follows:

$$
\begin{aligned}
& f_{0}(t)=W_{f}^{e}(0, t) \times \phi_{1}(t) \\
& f_{k}(t)=W_{f}^{e}(k, t) \times \psi_{k}(t)
\end{aligned}
$$

where $f(t)$ stands for the frequency-modulated amplitude-modulated signal. Accordingly, the empirical wavelet transform can achieve a self-adaptive decomposition of complex signals and obtain a series of frequency-modulated amplitude-modulated components, which are processed to obtain the instantaneous frequency and the instantaneous amplitude. 


\subsection{Analysis of the Simulation Signal}

In order to verify the algorithm, the analysis of simulation signal was performed. The mathematical expressions of the simulation signal are as follows:

$$
\begin{aligned}
& f_{1}(t)=6 t^{2} \\
& f_{2}(t)=\cos \left(10 \pi t+10 \pi t^{2}\right) \\
& f_{3}(t)=\left\{\begin{array}{c}
\cos (80 \pi t-15 \pi), t>0.5 \\
\cos (60 \pi t), \text { otherwise }
\end{array}\right. \\
& f(t)=f_{1}(t)+f_{2}(t)+f_{3}(t)
\end{aligned}
$$

where $f_{1}(t) f_{2}(t) f_{3}(t)$ represent the components at three different frequencies, and $f(t)$ represents the composite signal consisting of the first three signals. The EMD method and EWT method were used to process the simulation signal. The used simulation signal and its frequency spectrum are presented in Figures 1 and 2, respectively. In addition, the obtained decomposition results of the EWT and EMD are shown in Figures 3 and 4, respectively.
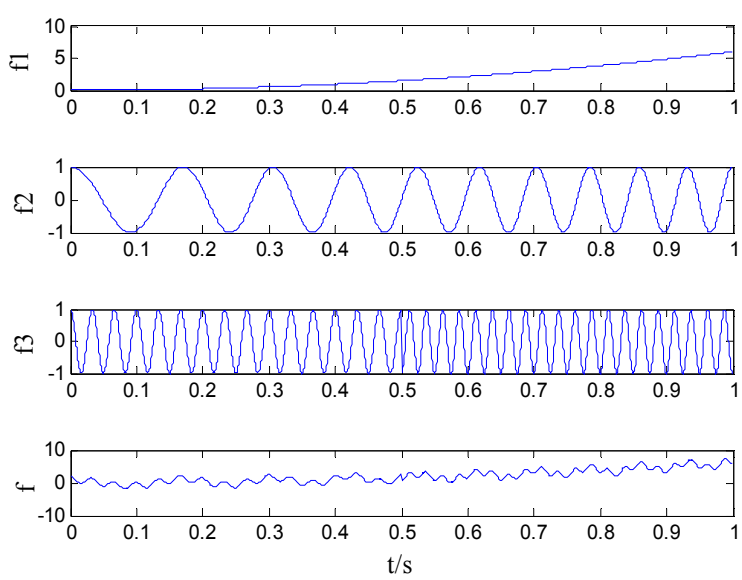

Figure 1. The simulated signal with three components.

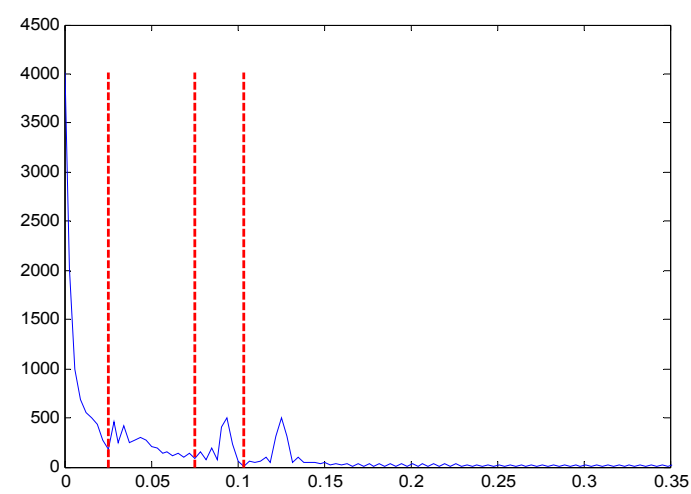

Figure 2. Spectrum and support boundary of the simulated signal.

As it can be seen in Figure 3, EWT separated the simulation signal into four signals with different frequencies, which can be considered as different modes since they have different frequencies and energies. However, in Figure 4, the modes are not decomposed, and both redundant information and false information are present. Accordingly, it is clear that EWT can decompose the signal more effectively and separate components of different frequencies more accurately than EMD. 

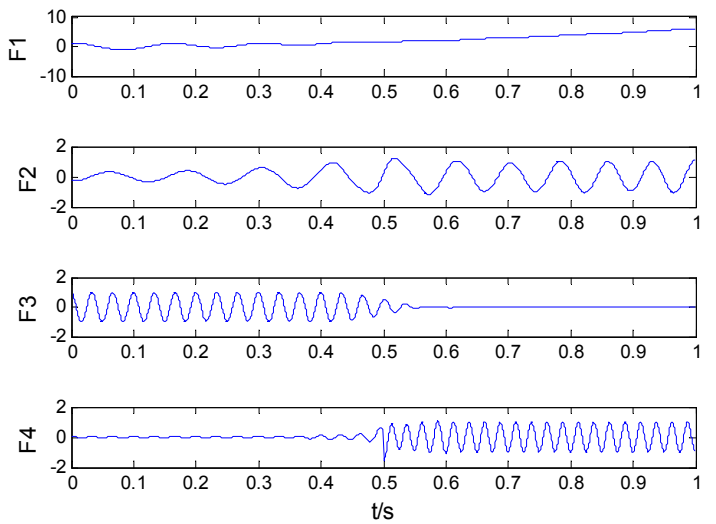

Figure 3. EWT result of simulated signal.
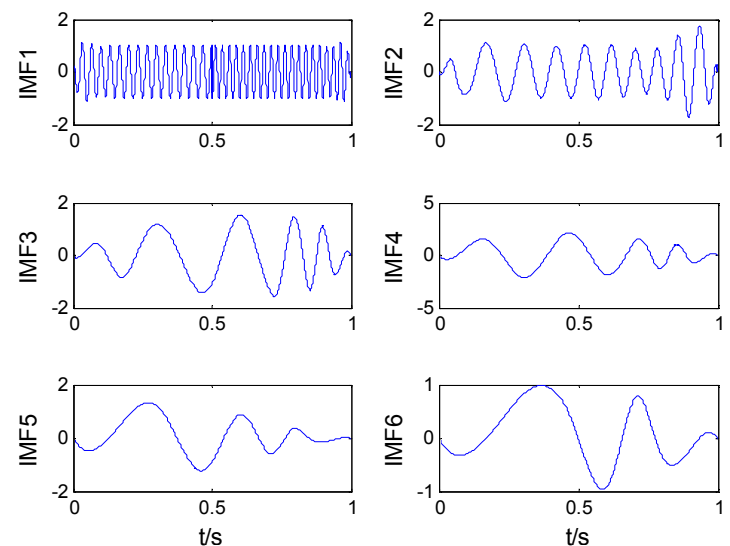

Figure 4. EMD result of the simulated signal.

\section{Ambiguity Correlation Classification}

\subsection{Ambiguity Correlation Theory}

Due to the advantages of non-stationary signal analysis, the ambiguity functions are widely used in engineering applications, such as radar signal analysis and optical information processing [31]. The mathematical expression of ambiguity functions is as follows:

$$
\begin{gathered}
A(\tau, \theta)=\frac{1}{2 \pi} \int r_{x}(t, \tau) e^{j \theta t} d t \\
r_{x}(t, \tau)=x(t+\tau / 2) x^{*}(t-\tau / 2)
\end{gathered}
$$

where $x(t)$ is the signal and $r_{x}(t, \tau)$ is the self-correlation function of signal, $A(\tau, \theta)$ is the ambiguity function.

The correlation coefficient method can be used to measure the similarity between two variables. The greater the correlation coefficient, the higher the correlation between variables. Therefore, the ambiguity correlation classifier is proposed in this paper based on the advantages of the correlation coefficient and ambiguity function. The combination of these two methods reduces the amount of calculations and avoids the interference from cross terms of the ambiguity function. The specific algorithm is as follows: 
(1) The correlation functions of ambiguity function images of two signals, namely $x(t), y(t)$, are calculated:

$$
R_{x y}(\tau, \theta)=\max _{\tau_{0}, \theta_{0}}\left|\int_{-\infty}^{+\infty} \int_{-\infty}^{+\infty} A_{x}(\tau, \theta) A_{y}\left(\tau-\tau_{0}, \theta-\theta_{0}\right) d \tau d \theta\right|
$$

where $R_{x y}(\tau, \theta)$ is a correlation function.

(2) The correlation functions are then used to normalize the correlation coefficient, and the corresponding mathematical expression is as follows:

$$
\rho_{x y}(\tau, \theta)=\frac{\max _{\tau_{0}, \theta 0}\left|\int_{-\infty}^{+\infty} \int_{-\infty}^{+\infty} A_{x}(\tau, \theta) A_{y}\left(\tau-\tau_{0}, \theta-\theta_{0}\right) d_{\tau} d \theta\right|}{\left[\int_{-\infty}^{+\infty} \int_{-\infty}^{+} A_{x}^{2}(\tau, \theta) d_{\tau} d_{\theta} \int_{-\infty}^{+\infty} \int_{-\infty}^{+} A_{y}^{2}(\tau, \theta) d_{\tau} d_{\theta}\right]^{\frac{1}{2}}}
$$

where $\rho_{x y}(\tau, \theta)$ is the correlation coefficient.

(3) When $\tau=0$ or $\theta=0$, the correlation coefficients are defined by:

$$
\begin{array}{r}
\rho_{x y}(0, \theta)=\frac{\max _{\tau_{0}, \theta 0}\left|\int_{-\infty}^{+\infty} \int_{-\infty}^{+\infty} A_{x}(0, \theta) A_{y}\left(0-\tau_{0}, \theta-\theta_{0}\right) d_{\tau} d \theta\right|}{\left[\int_{-\infty}^{+\infty} \int_{-\infty}^{+} A_{x}^{2}(0, \theta) d_{\tau} d_{\theta} \int_{-\infty}^{+\infty} \int_{-\infty}^{+} A_{y}^{2}(0, \theta) d_{\tau} d_{\theta}\right]^{\frac{1}{2}}} \\
\rho_{x y}(\tau, 0)=\frac{\max _{\tau_{0}, \theta 0}\left|\int_{-\infty}^{+\infty} \int_{-\infty}^{+\infty} A_{x}(\tau, 0) A_{y}\left(\tau-\tau_{0}, 0-\theta_{0}\right) d_{\tau} d \theta\right|}{\left[\int_{-\infty}^{+\infty} \int_{-\infty}^{+} A_{x}^{2}(\tau, 0) d_{\tau} d_{\theta} \int_{-\infty}^{+\infty} \int_{-\infty}^{+} A_{y}^{2}(\tau, 0) d_{\tau} d_{\theta}\right]^{\frac{1}{2}}}
\end{array}
$$

(4) Finally, the ambiguity correlation coefficient is calculated by:

$$
\bar{\rho}=\sqrt{\frac{\rho_{x y}^{2}(0, \theta)+\rho_{x y}^{2}(\tau, 0)}{2}}
$$

where $\bar{\rho}$ is the ambiguity correlation coefficient.

\subsection{Basic Principle of the Classifier}

The ambiguity correlation classifier represents a one-to-one classifier. Firstly, the signal is decomposed with the empirical wavelet transform decomposition method. Then, the mutual information method is proposed for the de-noising process, and the noiseless component is reconstructed in order to obtain the de-noising signal. Afterwards, the ambiguity correlation functions of three de-noised signal types are calculated, i.e., ambiguity functions of the class $A$ signal, test signal $C$, and the class $B$ signal. Then, the correlation coefficient is calculated using the ambiguity function of the reconstructed signal. The correlation coefficients of the class $A$ signal and test signal $C$ are labeled as $I$. The correlation coefficients of the class $B$ signal and test signal $C$ are labeled as II. Further, the correlation coefficients $I$ and $I I$ are compared, and if $I$ is larger, then $C$ belongs to class $A$; otherwise, $C$ belongs to class $B$. Similarly, this principle can be used to determine the signal collected in the next time moment. The principle of ambiguity correlation classifier is shown in Figure 5. 


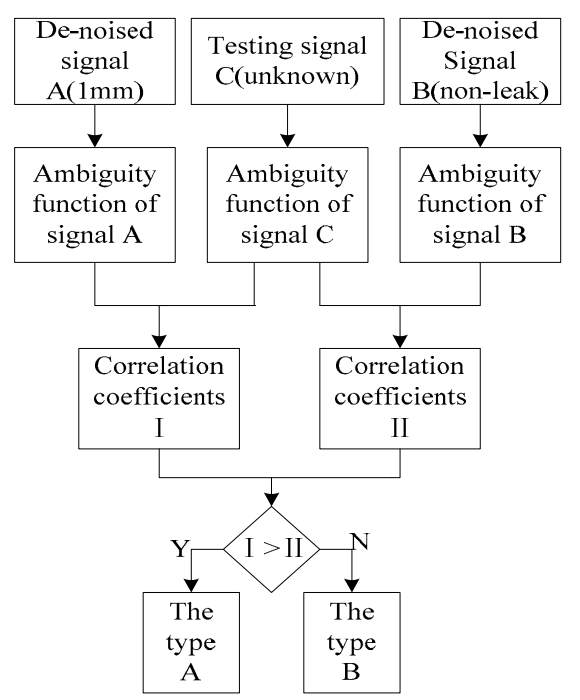

Figure 5. Classification flowchart.

\section{Bearing Faults Diagnosis Method}

The bearing faults diagnosis method based on EWT and ACC eliminates noise interference, extracts characteristic components from weak signals, and inputs reconstructed signals into the classifier in order to realize bearing fault detection. The specific steps are as follows:

(1) Collect the vibration signals and decompose these signals using EWT to derive multiple F components.

(2) Select noiseless components according to the mutual information.

(3) Reconstruct chosen noiseless components and inputs using ACC.

(4) Collect several groups of data for training and testing, and realize the fault diagnosis of rolling element bearings.

\section{Experimental Research}

\subsection{Collection of Experimental Data}

In this work, data from Case Western Reserve University were used for analysis, and the acoustic emission technique was used for detection. The basic layout of the test rig is shown in Figure 6 . It consisted of a 2 HP Reliance electric motor (Cleveland, OH, USA) that drove a shaft on which a torque transducer and encoder were mounted. Torque was applied to the shaft via a dynamometer and electronic control system. Further details about the test setup can be found in [31,32].

During the test process, the diameter of faults was from 0.007 to 0.028 in $(0.18-0.71 \mathrm{~mm})$ and faults were seeded on the drive-end and fan-end bearings (SKF deep-groove ball bearings: 6205-2RSJEM and 6203-2RSJEM, respectively) of the motor using an electro-discharge machining (EDM) (Cleveland, OH, USA). The faults were also seeded on the rolling elements and on the inner and outer races, and each faulty bearing was reinstalled (separately) on the test rig, which was then run at constant speed with the motor set to 0-3 horsepower (approximate motor speed was 1797-1720 rpm). The relevant bearing details and fault frequencies are presented in Table 1. During each test, acceleration was measured perpendicularly on the housing of the drive-end bearing (DE), and in some tests acceleration was also measured perpendicularly on the fan-end bearing housing (FE) and on the motor supporting base plate (BA). The length of collected data, $N$, was 3500 . 


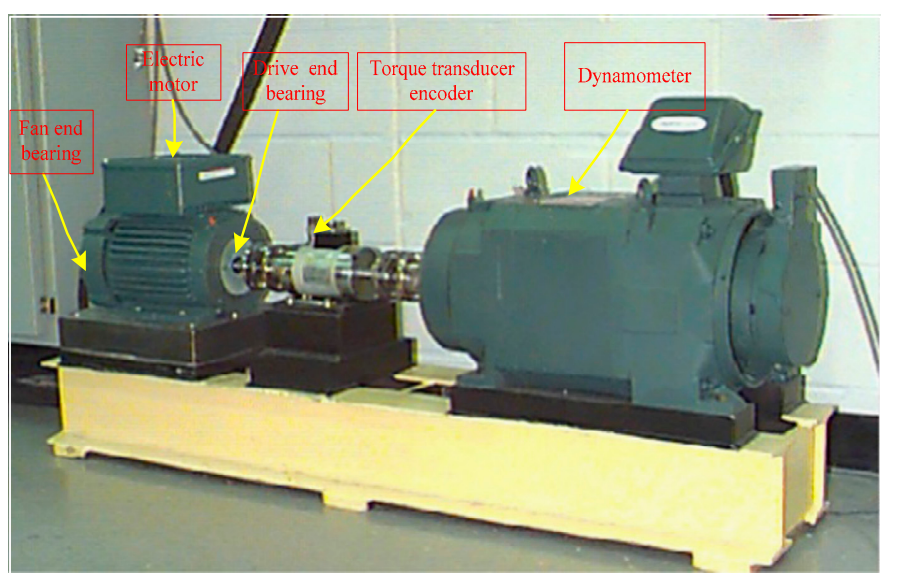

Figure 6. Field test acquisition device.

Table 1. Bearing details and fault frequencies.

\begin{tabular}{ccccc}
\hline \multirow{2}{*}{ Position on the Rig } & \multirow{2}{*}{ Model Number } & \multicolumn{2}{c}{ Fault Frequencies (Multiple of Shaft Speed) } \\
\cline { 3 - 5 } & & Outer Race & Inner Race & Rolling Element Ball \\
\hline Drive end & SKF6205-2RSJEM & 23.585 & 15.415 & 9.357 \\
Fan end & SKF6203-2RSJEM & 21.053 & 14.947 & 11.994 \\
\hline
\end{tabular}

\subsection{Analysis of Experimental Data}

The normal signal, the outer race fault signal, and the inner race fault signal, as shown in Figure 7, were used in the experiments. The outer race fault signal of the rolling element bearing was subjected to EWT decomposition, as shown in Figure 8.

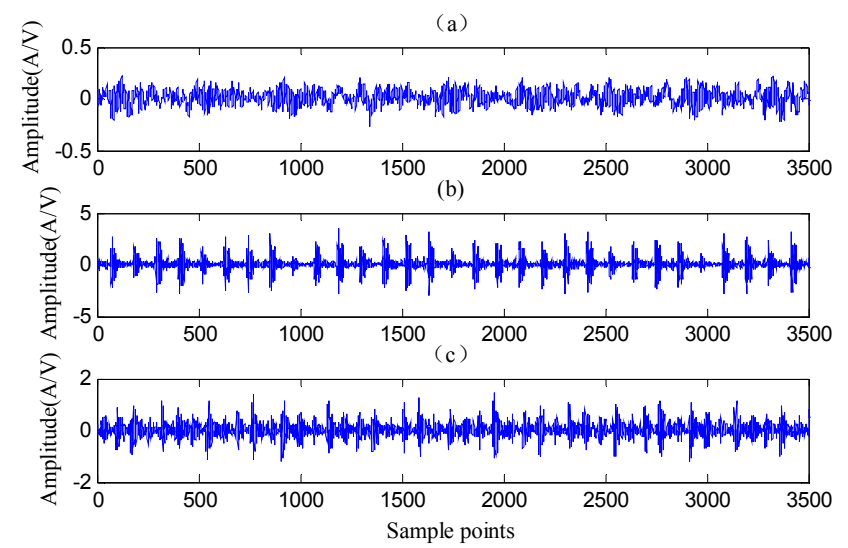

Figure 7. The vibration signals collected by sensor: (a) normal signal; (b) outer race fault signal; and (c) inner race fault signal.

As it can be seen in Figure 8, six components of the outer race fault signal were obtained by EWT decomposition. Some of the decomposed components were sensitive to fault information, while others were not correlated with faults. Thus, these $\mathrm{F}$ components needed to be processed further in order to extract the de-noising components and to reject the noise interference. The mutual information was a measure of statistical correlation between two random variables [33]. The higher the correlation between two random variables, the greater the mutual information. Thus, the de-noising components were extracted using the mutual information. Namely, for any two random variables, $x$ and $y$, the mutual information $I(X, Y)$ can be expressed by: 


$$
I(x, y)=\sum_{x, y} p_{x y}(x, y) \log \frac{p_{x y}(x, y)}{p_{x}(x) p_{y}(y)}
$$

where $p_{x y}(x, y)$ is joint distribution, $p_{x}(x)$ is marginal distribution, and $I(x, y)$ is the mutual information.
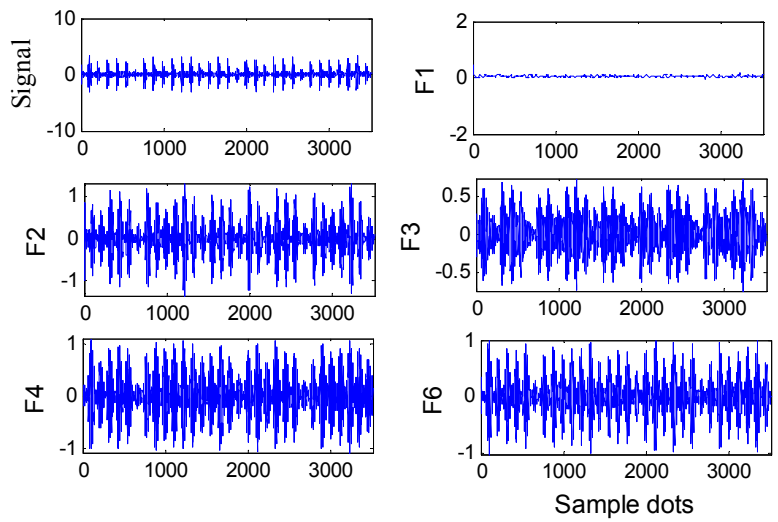

Figure 8. EWT results for the outer race fault signal.

Using Equation (20), the mutual information of decomposed F components and the original signal was calculated respectively, Figure 9.

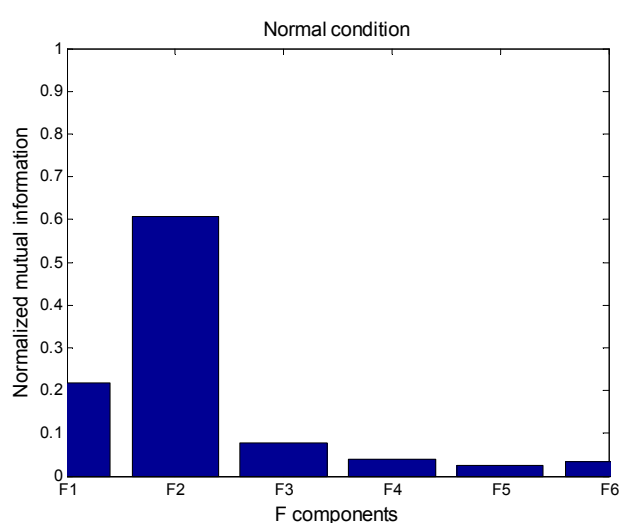

(a)

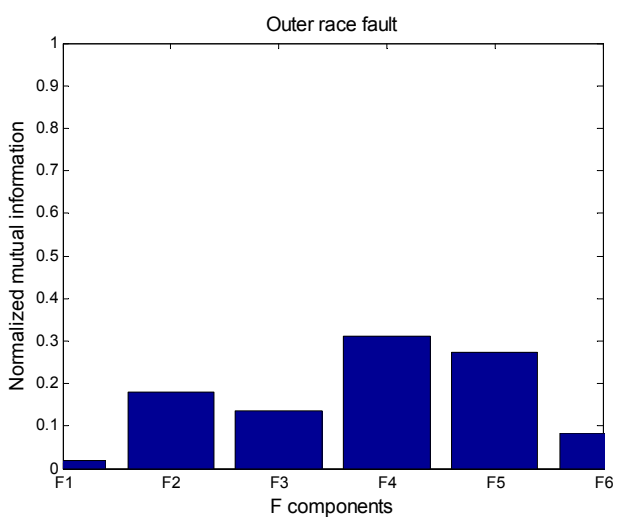

(b)

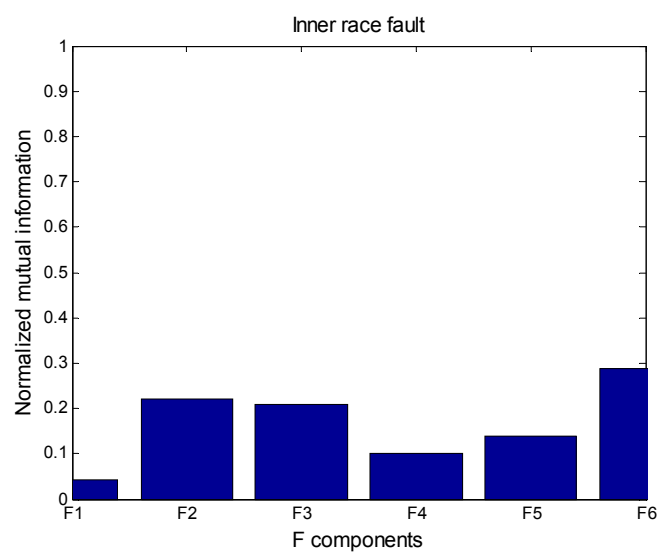

(c)

Figure 9. The mutual information of $F$ components for different conditions for: (a) normal signal; (b) outer race fault signal; and (c) inner race fault signal. 
Three different types of vibration signal were decomposed. The mutual information values of decomposed components and original signal were calculated. As it can be seen in Figure 9, in the case of the normal signal, the mutual information values of the first two components and original signal were larger than the others; thus, they were selected for reconstruction of the normal signal. In the case of the outer race fault signal, the mutual information values of components $F_{4}$ and $F_{5}$ were the greatest; thus, they were selected for reconstruction of the outer ring fault signal. Similarly, in the case of the inner ring fault signal, components $\mathrm{F}_{2}$ and $\mathrm{F}_{3}$ were selected for reconstruction. Consequently, the ambiguity correlation coefficients of the bearing reconstructed signal for different operative conditions were calculated.

\subsection{Ambiguity Correlation Classification}

The mean and standard deviation of the ambiguity correlation coefficient of the signals processed by EWT and EMD-based de-noising were calculated, and they are presented in Tables 2 and 3 , respectively.

Table 2. The mean and standard deviation for EMD.

\begin{tabular}{ccccc}
\hline \multicolumn{2}{c}{ Different Class Number } & \#1 & \#2 & $\# \mathbf{3}$ \\
\hline $\begin{array}{c}\text { different } \\
\text { operative conditions }\end{array}$ & $\begin{array}{c}\text { normal } \\
\text { state }\end{array}$ & $\begin{array}{c}\text { outer race } \\
\text { fault }\end{array}$ & $\begin{array}{c}\text { inner race } \\
\text { fault }\end{array}$ \\
\hline normal & mean & 0.5074 & 0.4664 & 0.4042 \\
state & standard deviation & 0.0630 & 0.0719 & 0.0853 \\
\hline outer race & mean & 0.4664 & 0.2415 & 0.3650 \\
fault & standard deviation & 0.0719 & 0.0540 & 0.0710 \\
\hline inner race & mean & 0.4042 & 0.3650 & 0.2789 \\
fault & standard deviation & 0.0853 & 0.0710 & 0.0552 \\
\hline
\end{tabular}

Table 3. The mean and standard deviation for EWT.

\begin{tabular}{ccccc}
\hline \multicolumn{2}{c}{ Different Class Number } & $\# \mathbf{1}$ & \#2 & $\# \mathbf{3}$ \\
\hline $\begin{array}{c}\text { different } \\
\text { operative conditions }\end{array}$ & $\begin{array}{c}\text { normal } \\
\text { state }\end{array}$ & $\begin{array}{c}\text { outer race } \\
\text { fault }\end{array}$ & $\begin{array}{c}\text { inner race } \\
\text { fault }\end{array}$ \\
\hline normal & mean & 0.6347 & 0.3134 & 0.1025 \\
state & standard deviation & 0.0395 & 0.1164 & 0.0969 \\
\hline outer race & mean & 0.3134 & 0.5407 & 0.0685 \\
fault & standard deviation & 0.1164 & 0.0240 & 0.0543 \\
\hline inner race & mean & 0.1025 & 0.0685 & 0.2253 \\
fault & standard deviation & 0.0969 & 0.0534 & 0.0452 \\
\hline
\end{tabular}

As it is presented in Table 2, the mode mixing that is present in the empirical modal decomposition process leads to illusive component decomposition. Namely, the reconstructed signal obtained using the mutual information contained a noise component. Therefore, there was no significant difference in the mean of the correlation coefficients of the three decomposed signal types in the ambiguity domain; thus, it was impossible to monitor the rolling element bearings. On the other hand, when EWT was used to process the signals in order to obtain the inherent modal component of the signal and to remove the noise interference, the mean of the correlation coefficients of three signal types in the ambiguity domain could be clearly distinguished (Table 3). With the aim to provide a visual comparison of the obtained results, the mean and standard deviation of the ambiguity correlation coefficients presented in Tables 2 and 3 are plotted in Figures 10 and 11, respectively. 


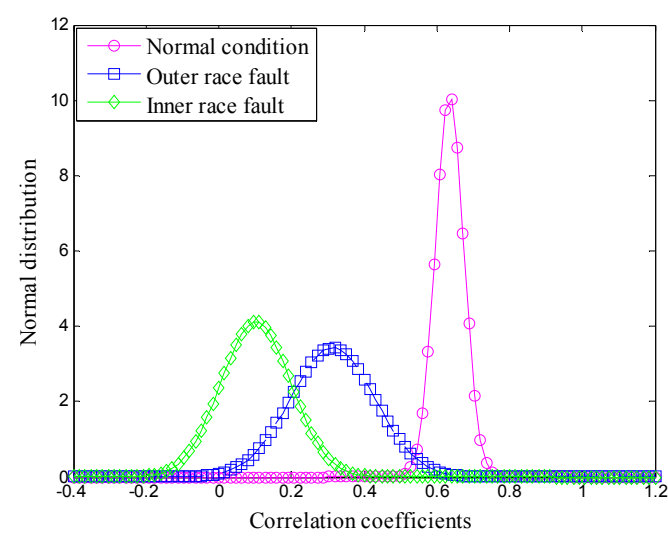

(a)

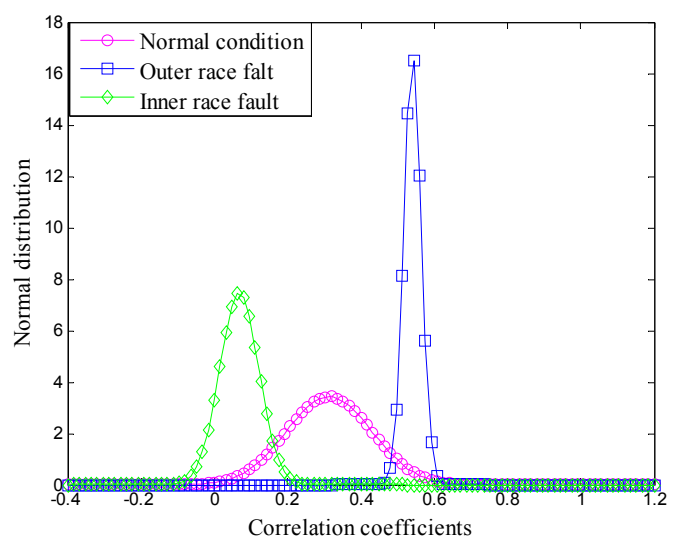

(b)

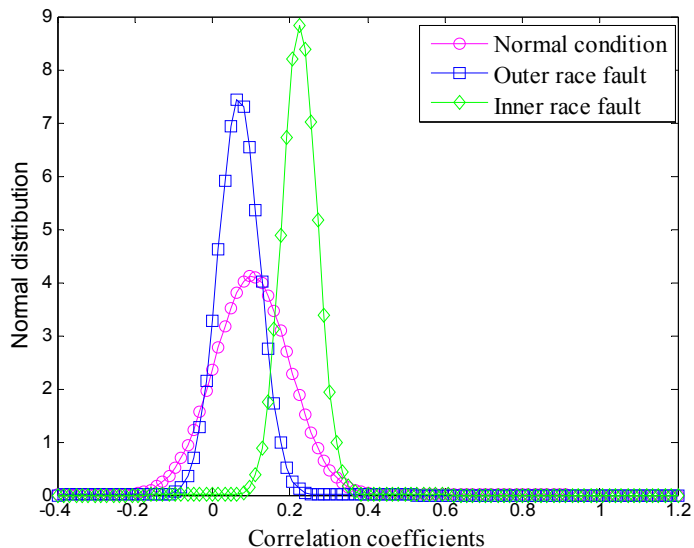

(c)

Figure 10. Normal distribution of EWT: (a) \#1; (b) \#2; and (c) \#3.

As it is shown in Figures 10 and 11, the normal distribution curves visualize the diagnosis of three operative conditions of rolling bearings and provide the real-time monitoring. Moreover, there are no significant differences between the normal distribution curves of the EMD-based correlation coefficients. In contrast to EMD decomposition, EWT processes the signal within the wavelet framework, so it can extract an inherent signal mode without mode mixing. Hence, the EWT-based method can distinguish between three modes, and the differences between the normal distribution curves of the correlation coefficients in the ambiguity domain are significant.

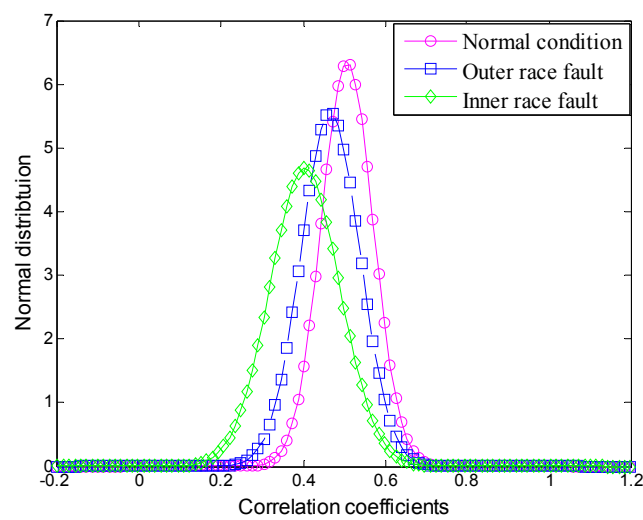

(a)

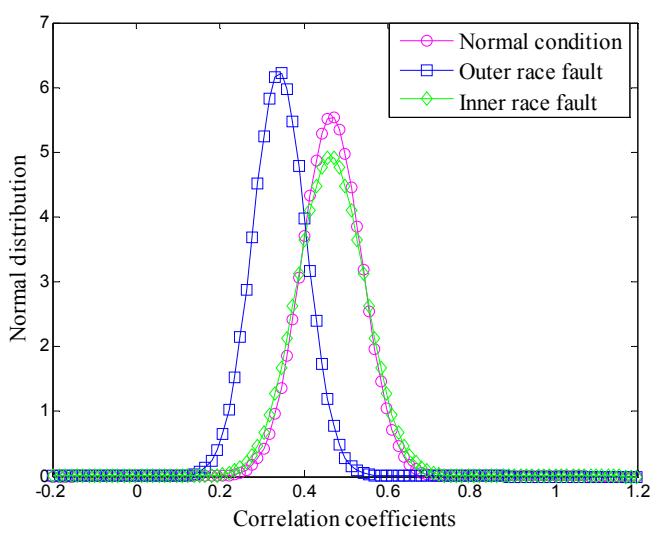

(b)

Figure 11. Cont. 


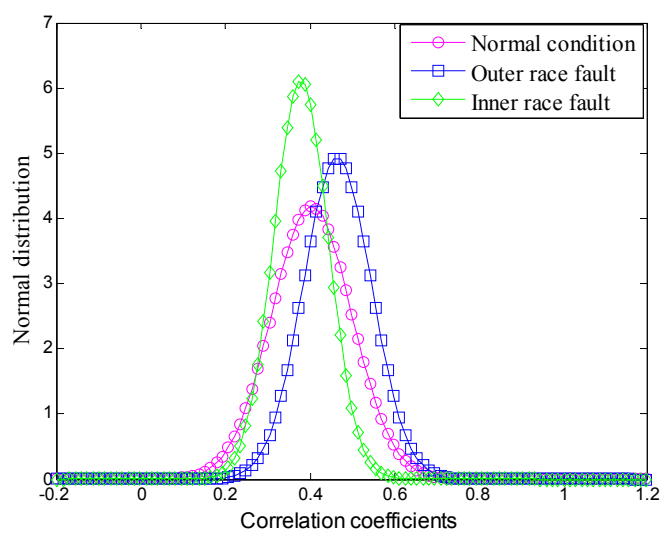

(c)

Figure 11. Normal distribution of EMD: (a) \#1; (b) \#2; and (c) \#3.

As it was stated above, 20 datasets were used for training and testing, namely 15 datasets were used for training, and the remaining five datasets were used for testing. The classification results and accuracies are presented in Figure 12.

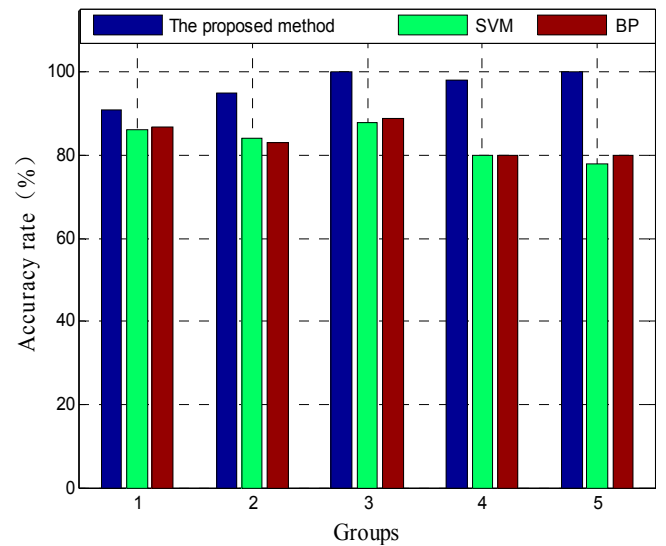

(a)

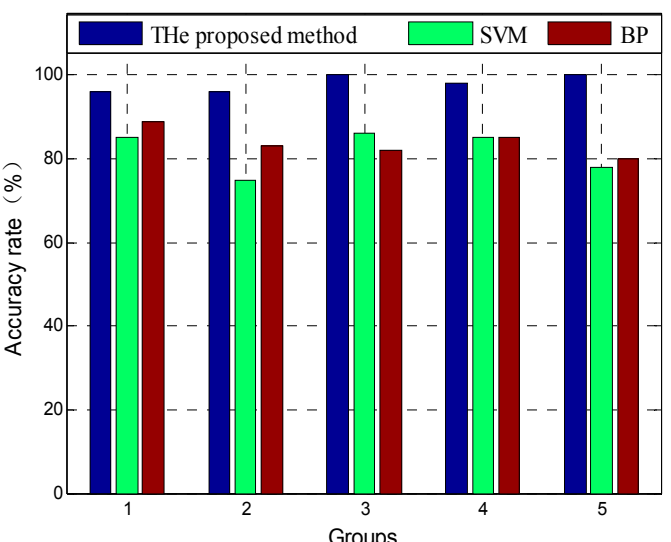

(b)

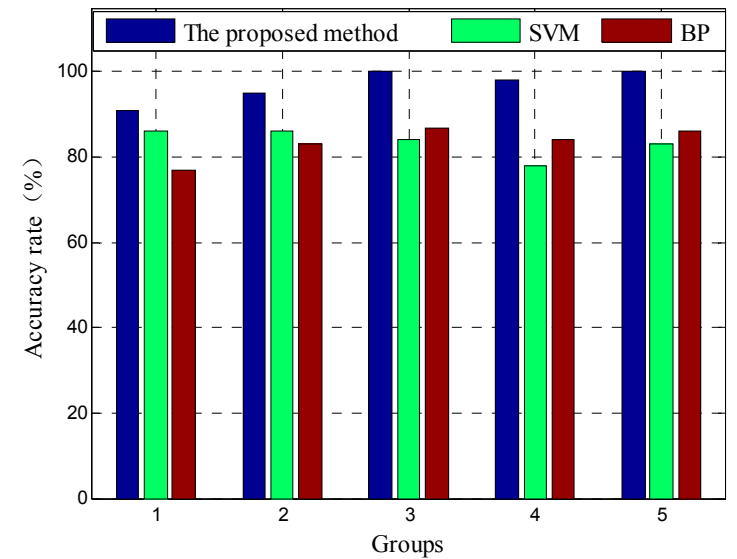

(c)

Figure 12. The classification result of three classifiers: (a) proposed method; (b) SVM; and (c) BP.

According to the obtained test results presented in Figure 12, the proposed classifier has a shorter training time, simpler calculation, higher identification rate, and higher ability to diagnose different operative conditions of rolling bearings than BP and SVM classifiers. 


\section{Conclusions}

The collected vibration signals are often mixed with substantial ambient noise, which makes the fault signal features insignificant for the rolling bearings fault diagnosis. Thus, a novel fault diagnosis method for rolling element bearings based on EWT and ACC is proposed. Due to mode mixing in the EMD decomposition, in the proposed method EWT is used to decompose the vibration signal in order to obtain the accurate components and to eliminate the mode mixing. Moreover, in order to address the disadvantages of traditional BP and SVM classifiers, such as complex parameter setting and low convergence rates, the ambiguity functions and correlation coefficients are combined to achieve the ambiguity correlation classification. The ambiguity correlation classifier is based on a simple calculation process and requires no parameter setting. The proposed method was verified by experiments, and the experimental results have indicated that the proposed classifier can monitor different operative conditions of rolling bearings with higher identification rates than BP and SVM.

Acknowledgments: This work was supported by the key project of China Spark Program (2015GA750005) and Key Science and Technology Program of Henan Province, China (152102210130).

Author Contributions: Xingmeng Jiang and Li Wu conceived and designed the experiments; Xingmeng Jiang performed the experiments and analyzed the experimental data; $\mathrm{Li} \mathrm{Wu}$ and Mingtao Ge provided guidance and recommendations for research; and, lastly, Xingmeng Jiang contributed to the contents and writing of the manuscript. All authors have read and approved the final manuscript.

Conflicts of Interest: The authors declare no conflict of interest.

\section{References}

1. Lei, Y.G.; Zuo, M.J. Fault diagnosis of rotating machinery using an improved HHT based on EEMD and sensitive IMFs. Meas. Sci. Technol. 2009, 20, 1-12. [CrossRef]

2. Zhang, F.; Liu, Y.; Chen, C.; Li, Y.-F.; Huang, H.-Z. Fault diagnosis of rotating machinery based on kernel density estimation and Kullback-Leibler divergence. J. Mech. Sci. Technol. 2014, 11, 4441-4454. [CrossRef]

3. Vidal, Y.; Tutivén, C.; Rodellar, J.; Acho, L. Fault diagnosis and fault-tolerant control of wind turbines via a discrete time controller with a disturbance compensator. Energies 2015, 8, 4300-4316. [CrossRef]

4. Rafiee, J.; Rafiee, M.A.; Tse, P.W. Application of mother wavelet functions for automatic gear and bearing fault diagnosis. Expert Syst. Appl. 2010, 37, 4568-4579. [CrossRef]

5. Morhain, A.; Mba, D. Bearing defect diagnosis and acoustic emission. Proc. Inst. Mech. Eng. Part J J. Eng. Tribol. 2003, 217, 257-272. [CrossRef]

6. Yen, C.L.; Lu, M.C.; Chen, J.L. Applying the self-organization feature map (SOM) algorithm to AE-based tool wear monitoring in micro-cutting. Mech. Syst. Signal Process. 2013, 34, 353-366. [CrossRef]

7. Feng, Z.; Zuo, M.J.; Qu, J.; Tian, T.; Liu, Z. Joint amplitude and frequency demodulation analysis based on local mean decomposition for fault diagnosis of planetary gearboxes. Mech. Syst. Signal Process. 2013, 40, 56-75. [CrossRef]

8. Lin, J.; Qu, L. Feature extraction based on Morlet wavelet and its application for mechanical fault diagnosis. J. Sound Vib. 2000, 234, 135-148. [CrossRef]

9. Nandi, S.; Toliyat, H.A.; Li, X. Condition monitoring and fault diagnosis of electrical motors-A review. IEEE Trans. Energy Convers. 2005, 20, 719-729. [CrossRef]

10. Lopez-Ramirez, M.; Ledesma-Carrillo, L.; Cabal-Yepez, E.; Rodriguez-Donate, C.; Miranda-Vidales, H.; Garcia-Perez, A. EMD-Based Feature Extraction for Power Quality Disturbance Classification Using Moments. Energies 2016, 9, 565. [CrossRef]

11. Lynagh, N.; Rahnejat, H.; Ebrahimi, M.; Aini, R. Bearing induced vibration in precision high speed routing spindles. Int. J. Mach. Tool Manuf. 2000, 40, 561-577. [CrossRef]

12. Vafaei, S.; Rahnejat, H. Indicated repeatable runout with wavelet decomposition (IRR-WD) for effective determination of bearing-induced vibration. J. Sound Vib. 2003, 260, 67-82. [CrossRef]

13. Wardle, F.P. Vibration forces produced by waviness of the rolling surfaces of thrust loaded ball bearings Part 1: Theory. Proc. Inst. Mech. Eng. Part C J. Mech. Eng. Sci. 1988, 202, 305-312. [CrossRef]

14. Chen, S.W.; Chen, Y.H. Hardware design and implementation of a wavelet de-noising procedure for medical signal processing. Sensors 2015, 15, 26396-26414. [CrossRef] [PubMed] 
15. Zhong, J.H.; Wong, P.K.; Yang, Z.X. Simultaneous-Fault Diagnosis of Gearboxes Using Probabilistic Committee Machine. Sensors 2016, 16, 185. [CrossRef] [PubMed]

16. Xiao, Q.; Li, J.; Bai, Z.; Sun, J.; Zhou, N.; Zeng, Z. A Small Leak Detection Method Based on VMD Adaptive De-Noising and Ambiguity Correlation Classification Intended for Natural Gas Pipelines. Sensors 2016, 16, 2116. [CrossRef] [PubMed]

17. Wang, T.; Zhang, M.; Yu, Q.; Zhang, H. Comparing the applications of EMD and EEMD on time-frequency analysis of seismic signal. J. Appl. Geophys. 2012, 83, 29-34. [CrossRef]

18. Wang, W.; Chau, K.; Xu, D.; Chen, X.-Y. Improving forecasting accuracy of annual runoff time series using ARIMA based on EEMD decomposition. Water Resour. Manag. 2015, 29, 2655-2675. [CrossRef]

19. Sun, J.; Xiao, Q.; Wen, J.; Wang, F. Natural gas pipeline small leakage feature extraction and recognition based on LMD envelope spectrum entropy and SVM. Measurement 2014, 55, 434-443. [CrossRef]

20. Gilles, J. Empirical wavelet transforms. IEEE Trans. Signal Process. 2013, 61, 3999-4010. [CrossRef]

21. Chen, J.; Li, Z.; Pan, J.; Chen, G.; Zi, Y.; Yuan, J.; Chen, B.; He, Z. Wavelet transform based on inner product in fault diagnosis of rotating machinery: A review. Mech. Syst. Signal Process. 2016, 70, 1-35. [CrossRef]

22. Chen, J.; Pan, J.; Li, Z.; Zi, Y.; Chen, X. Generator bearing fault diagnosis for wind turbine via empirical wavelet transform using measured vibration signals. Renew. Energy 2016, 89, 80-92. [CrossRef]

23. Hu, J.; Wang, J. Short-term wind speed prediction using empirical wavelet transform and Gaussian process regression. Energy 2015, 93, 1456-1466. [CrossRef]

24. Maheshwari, S.; Pachori, R.B.; Acharya, U.R. Automated diagnosis of glaucoma using empirical wavelet transform and correntropy features extracted from fundus images. IEEE J. Biomed. Health 2016, 21, 803-813. [CrossRef] [PubMed]

25. Jiang, Y.; Zhu, H.; Li, Z. A new compound faults detection method for rolling bearings based on empirical wavelet transform and chaotic oscillator. Chaos Soliton Fractals 2016, 89, 8-19. [CrossRef]

26. Pérez-Ortiz, M.; Jiménez-Fernández, S.; Gutiérrez, P.A.; Alexandre, E.; Hervás-Martínez, C.; Salcedo-Sanz, S. A Review of Classification Problems and Algorithms in Renewable Energy Applications. Energies 2016, 9 , 607. [CrossRef]

27. Riahi, M.; Shamekh, H.; Khosrowzadeh, B. Differentiation of leakage and corrosion signals in acoustic emission testing of aboveground storage tank floors with artificial neural networks. Russ. J. Nondestruct. Test. 2008, 44, 436-441. [CrossRef]

28. Lu, J.; Huang, J.; Lu, F. Sensor Fault Diagnosis for Aero Engine Based on Online Sequential Extreme Learning Machine with Memory Principle. Energies 2017, 10, 39. [CrossRef]

29. Yang, B.S.; Han, T.; An, J.L. ART-KOHONEN neural network for fault diagnosis of rotating machinery. Mech. Syst. Signal Process. 2004, 18, 645-657. [CrossRef]

30. Xiao, Y.; Kang, N.; Hong, Y.; Zhang, G. Misalignment Fault Diagnosis of DFWT Based on IEMD Energy Entropy and PSO-SVM. Entropy 2017, 19, 6. [CrossRef]

31. Kutyniok, G. Ambiguity functions, Wigner distributions and Cohen's class for LCA groups. J. Math. Anal. Appl. 2003, 277, 589-608. [CrossRef]

32. Smith, W.A.; Randall, R.B. Rolling element bearing diagnostics using the Case Western Reserve University data: A benchmark study. Mech. Syst. Signal Process. 2015, 64, 100-131. [CrossRef]

33. Kinney, J.B.; Atwal, G.S. Equitability, mutual information, and the maximal information coefficient. Proc. Natl. Acad. Sci. USA 2014, 111, 3354-3359. [CrossRef] [PubMed]

(C) 2017 by the authors. Licensee MDPI, Basel, Switzerland. This article is an open access article distributed under the terms and conditions of the Creative Commons Attribution (CC BY) license (http:/ / creativecommons.org/licenses/by/4.0/). 